\title{
Treatment Patterns and Resource Utilization of Pregnant Women with Inflammatory Rheumatic Diseases or Psoriasis in Germany: A Claims Database Analysis
}

\author{
Katja Blaschke · Rebecca Fischer-Betz • Ursula Marschall • \\ Wojciech Dombrowsky • Lars Joeres • Tanja Heidbrede • \\ Ingrid Schubert
}

Received: April 23, 2021 / Accepted: July 5, 2021 / Published online: August 24, 2021

(C) The Author(s) 2021

\section{ABSTRACT}

Background: Uncontrolled inflammatory disease activity can impact pregnancy outcomes and the health of the mother and child. This retrospective claims database analysis assessed treatment patterns before, during, and after pregnancy among women with inflammatory rheumatic disease (IRD; axial spondyloarthritis [axSpA], psoriatic arthritis [PsA], and rheumatoid arthritis [RA]) or psoriasis (PSO) in Germany.

Methods: Data were extracted from the BARMER sickness fund (2013-2017). Pregnant women (18-45 years) with documented IRD or PSO

Supplementary Information The online version contains supplementary material available at https:// doi.org/10.1007/s40744-021-00347-3.

K. Blaschke $(\bowtie) \cdot$ I. Schubert

PMV Research Group, Faculty of Medicine and University Hospital Cologne, University of Cologne, Herderstraße 52, D-50931 Cologne, Germany e-mail: katja.blaschke@uk-koeln.de

R. Fischer-Betz

Department for Rheumatology and Hiller Research Institute, Heinrich Heine University Düsseldorf, Düsseldorf, Germany

U. Marschall

Department of Medicine and Health Services Research, BARMER Statutory Health Insurance Fund, Wuppertal, Germany

W. Dombrowsky · L. Joeres · T. Heidbrede UCB Pharma, Monheim, Germany diagnoses were compared with age-matched controls from the same database for the analysis of patient characteristics, healthcare resource utilization, and pharmacological treatment during pregnancy. Reported measures included the proportion of women with pharmacological prescriptions or hospitalization/new prescription of corticosteroids or biologics in the 180 days before pregnancy, during pregnancy, and 180 days after delivery. Pre-specified prescription categories (such as disease-specific drugs [not including biologics]) were identified by anatomical therapeutic chemical classification codes. Extrapolated values to the German statutory health insurance population are reported.

Results: Overall, 2702 pregnant women with IRD (axSpA: 1063; PsA: 660; RA: 979) and 6527 with PSO were identified. The proportion of women with IRD receiving prescriptions for disease-specific drugs reduced during pregnancy and remained stable after delivery (before: 15.0\%; during: 9.0\%; after: 9.7\%). The proportion of women with PSO receiving prescriptions for disease-specific drugs was low (before: 0.6\%; during: $0.3 \%$; after: $0.1 \%$ ). The proportion of women with hospitalization/new prescription of corticosteroids or biologics decreased during pregnancy, compared with pre-pregnancy, and increased after delivery in women with IRD (before: 9.0\%; during: 5.1\%; after: 11.1\%) and PSO (before: 3.5\%; during: 1.9\%; after: $2.7 \%$ ).

Conclusions: A reduction in pharmacological treatment during pregnancy was observed for 
women with IRD in Germany. Many women with IRD did not return to pre-pregnancy treatments after delivery, despite signs of disease exacerbation, such as hospitalization and initiation of treatment with corticosteroids/biologics, in this period.

Keywords: Axial spondyloarthritis; Claims data; Healthcare resource utilization; Inflammatory rheumatic disease; Pregnant women; Psoriasis; Psoriatic arthritis; Rheumatoid arthritis; Treatment patterns

\section{Summary Points}

\section{Why carry out this study?}

Uncontrolled inflammatory disease activity during pregnancy can impact pregnancy outcomes and the health of the mother and child after delivery.

At present, there is a lack of real-world data available on the treatment patterns of pregnant women with inflammatory rheumatic disease (IRD) and psoriasis (PSO), highlighting the requirement to better understand these women's unmet clinical needs.

This study aimed to assess treatment patterns and healthcare resource utilization before, during, and after pregnancy among women with IRD or PSO in Germany.

\section{What was learned from the study?}

Large proportions of women with IRD did not return to their pre-pregnancy treatments in the 180 days after delivery, despite signs of disease exacerbation, such as hospitalization and the initiation of treatment with corticosteroids/biologics, in this period.

The identification of potential unmet needs highlights that postpartum care should be prioritized for women with IRD and that this could lead to improved outcomes.

\section{INTRODUCTION}

Psoriasis (PSO) and inflammatory rheumatic diseases (IRDs), such as axial spondyloarthritis (axSpA), psoriatic arthritis (PsA), and rheumatoid arthritis (RA), can have a life-long negative impact on patients' quality of life [1-3]. The overlap of these chronic inflammatory diseases with reproductive age in women can pose treatment challenges due to the potential negative impacts of active disease and high-risk medications on conception, pregnancy outcomes, and breastfeeding $[4,5]$.

Uncontrolled disease activity and disease flares during pregnancy or after delivery pose the greatest risk to pregnancy outcomes, and the health of the mother and child after delivery [6]. Results from a Danish and Swedish national prevalence study showed that women with RA had higher rates of most adverse pregnancy outcomes compared with women without RA [7]. Furthermore, increased RA disease activity during pregnancy and active axSpA disease during the second trimester of pregnancy were shown to be associated with increased risk of preterm birth [8,9]. Similarly, results from a Danish and Swedish observational study showed that women with severe PSO had an increased risk of preterm birth and low birth weight, compared with women with non-severe PSO [10].

Although some studies indicate that PSO, PsA, and RA disease activity may improve during pregnancy [11-15], and deteriorate after pregnancy for patients with axSpA or ankylosing spondylitis (AS) $[16,17]$, the course of disease in individual patients is unpredictable. Furthermore, disease flares after delivery are frequently reported among women with rheumatic diseases [18]. Due to the association between uncontrolled disease activity and the health of the mother and child, patients with active disease during pregnancy require effective therapeutic interventions. Recent EULAR (European Alliance of Associations for Rheumatology) points to consider and ACR (American College of Rheumatology) guidelines report those therapeutics that are recommended to treat chronic rheumatic conditions 
before, during, and after pregnancy, including whilst breastfeeding [6, 19]. These guidelines support the effective management of patients; however, there remains insufficient evidence regarding the safety of some anti-rheumatic drugs during pregnancy or whilst breastfeeding [19]. There are currently no specific guidelines that cover the treatment of psoriasis before, during, and after pregnancy; however, recent American and European general treatment guidelines do report on the systemic therapies which can be taken by women with psoriasis during these periods [20-23].

At present, there is a lack of real-world data available on the treatment patterns of IRD and PSO in pregnant women, and further investigation of this will increase the understanding of these women's unmet clinical needs. Claims data provide longitudinal datasets that are particularly useful to provide insights into the patterns of treatment and disease over time (e.g., before, during, and after pregnancy) [24].

This study aims to use claims data from a large statutory health insurance fund in Germany, covering approximately 9 million insured persons [25], to assess treatment patterns and healthcare resource utilization (HCRU) over time in pregnant women with a diagnosis of IRD or PSO.

\section{METHODS}

\section{Database and Study Design}

This retrospective claims database analysis used data from the BARMER sickness fund Scientific Data Warehouse (Wissenschaftliches DataWareHouse [W-DWH]), from 2013 to 2017. Permission to use the W-DWH database was contracted between the PMV Research Group and BARMER. These data were pseudonymized for the researchers; however, information generated in different sectors of care could be linked using the study identifier to allow the longitudinal assessment of healthcare use. Ethical approval was not required for this study as the data analyzed were retrospective and were pseudonymized and anonymized, so that the subsequent assignment to individual patient data was impossible. Direct and project-specific access to the BARMER sickness fund W-DHW was contracted between BARMER and the PMV Research Group.

The study population comprised pregnant women aged 18-45 years who were continually insured for 1 year before pregnancy, during pregnancy, and the 180 days after delivery. Women with a calculated start of pregnancy between January 1, 2015 and August 31, 2016 and subsequent delivery (live birth, still birth, or preterm birth) during a hospital stay between 2015 and 2017 were included in the analysis; this allowed for a follow-up of 180 days after delivery within 2017 (Fig. 1).

Pregnant women with IRD (axSpA, PsA, and RA) or PSO were compared with pregnant women without a documented diagnosis of IRD or PSO, from the same database, for the analysis of patient characteristics, healthcare resource utilization, and pharmacological treatment during pregnancy. For this control group, 1:1 matching was conducted for age at the start of pregnancy and for the timing of the pregnancy (i.e., the calculated start of pregnancy had to happen in the same quarter of the year; Fig. 1). Control groups were determined separately for the axSpA, RA, PsA, and overall IRD populations, hence the IRD control group is not the sum of the axSpA, RA, and PsA control groups. The prescription of disease-specific drugs and biologics by physicians over time and the hospitalization/new prescription of corticosteroids or biologics were only investigated in pregnant women with IRD (axSpA, PsA, and RA) or PSO and not the control group.

\section{Study Definitions}

\section{IRD and PSO Diagnosis}

Diagnoses were coded by International Classification of Diseases (ICD)-10 codes (axSpA: M45; PsA: M07.0-M07.3, L40.5+; RA: M05.8, M06.0, M06.8; PSO: L40.0, L40.1, L40.3, L40.8, L40.9). An ICD-10 code for non-radiographic (nr-)axSpA was not available, therefore, patients with nr-axSpA are not represented separately in these analyses. Patients with IRD diagnoses were further defined as patients with at least 


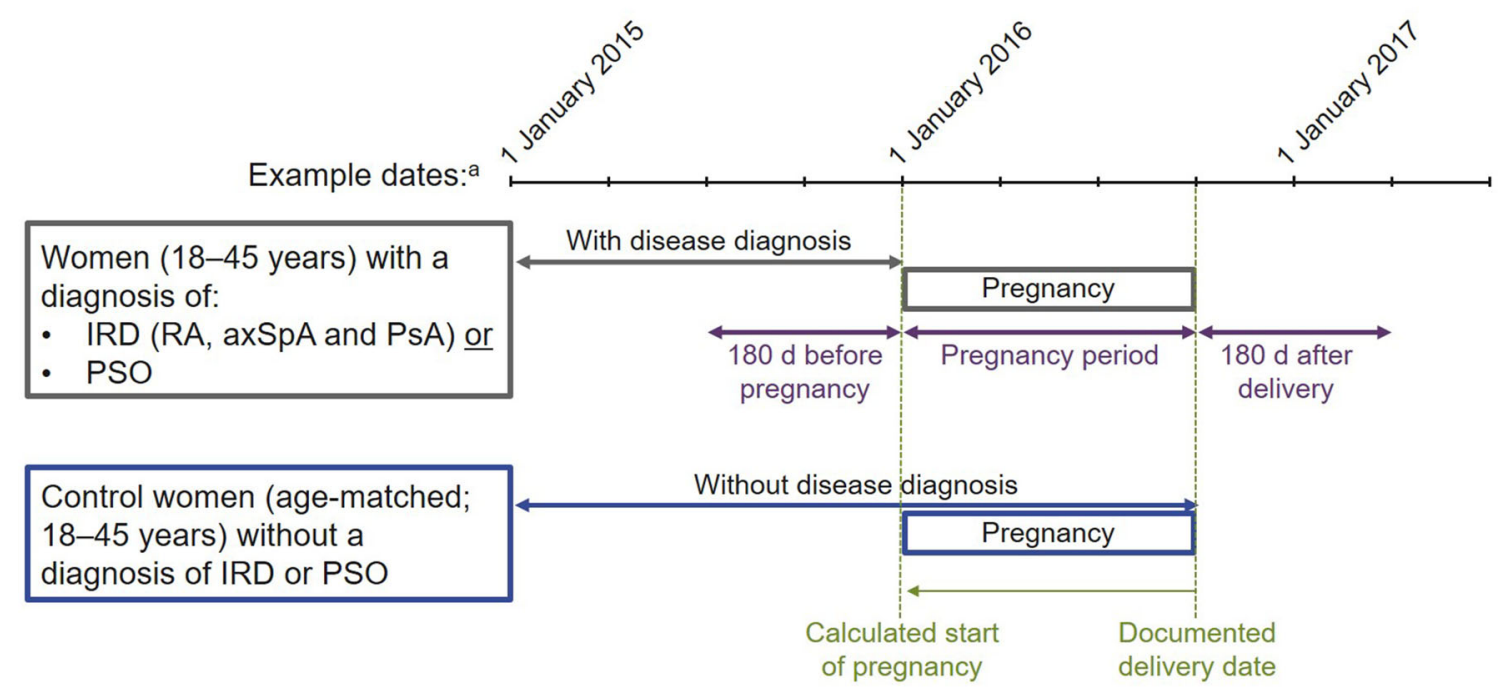

Fig. 1 Study design. ${ }^{\text {a }}$ The dates in this figure are descriptive to demonstrate the study design for one individual. Women with a calculated start of pregnancy between January 1, 2015 and August 31, 2016, and subsequent

one diagnosis of either axSpA, PsA, or RA. Women with a documented diagnosis of IRD or PSO had to fulfil one of the following criteria to be included in the analyses: documentation of either (1) a hospital discharge diagnosis, or (2) an assured outpatient diagnosis in at least two quarters of the year under observation (where the second diagnosis was used as the index diagnosis).

Study patients with multiple diagnoses were assigned as follows: patients with PSO and PsA codes were assigned to the PsA disease group, patients with axSpA, PSO, or PsA codes in addition to RA were assigned to the RA disease group, and patients with PSO or PsA codes in addition to axSpA were assigned to the axSpA disease group. This ensured the unique allocation of patients to one disease and, therefore, avoided the counting of patients more than once. Furthermore, these groupings take into account disease progression (PSO to PsA) and the most severe disease diagnoses (axSpA and RA).

\section{Pregnancy}

Women were identified by their hospital stay and the respective ICD-10, diagnosis-related delivery during a hospital stay between 2015 and 2017, were included in the analyses. axSpA axial spondyloarthritis, IRD inflammatory rheumatic diseases, $P_{S} A$ psoriatic arthritis, $P S O$ psoriasis, $R A$ rheumatoid arthritis

group (DRG), or operation and procedure classification system (OPS) codes for delivery (Supplementary Material: Table S1). One code from two of the ICD-10, DRG, or OPS groups had to be documented for women to be included in the analysis. Women with diagnoses of an abortion or miscarriage (including ectopic pregnancy) during the hospital stay were excluded (Supplementary Material: Table S2). Women with greater than one recorded delivery within 153 days were further investigated to verify the pregnancy. Those with an overlap of hospital stays or a subsequent hospital discharge and admission date were classed as having had one pregnancy; all other women with more than one coded delivery were excluded.

Estimated pregnancy start dates, defined as the first day of the last menstruation, were calculated using the date of delivery and the duration of pregnancy. Date of delivery was defined as the first date an OPS code of delivery was billed, if none was available the discharge date was used. Duration of pregnancy was documented by ICD-10 code O09 as a secondary diagnosis and was recorded in weekly periods, with the last-mentioned week of pregnancy used to calculate the start of pregnancy from the 
date of delivery. A maximum duration of 42 weeks was calculated since, according to German guidelines, induction of labor is indicated at this time [26]. The study population was restricted to women for whom information regarding the duration of pregnancy was reported.

The following time periods of pregnancy were investigated: 180 days before pregnancy, during pregnancy, and 180 days after delivery. Due to quarterly billing of outpatient claims, all outpatient diagnoses or physician contacts were recorded if the billing quarter extended into one of the time periods of pregnancy.

\section{Physician Contact}

Physicians were identified by their lifelong physician numbers (Lebenslange Arztnummer [LANR]), which were pseudonymized for the study. The following specialist physician groups were included in this study and were identified from the LANRs: general practitioner (GP) and internal medicine specialist working as GP (LANR codes: 01, 02, 03), internal medicine specialist (LANR code: 23), internal medicine and rheumatology specialist (LANR code: 31), gynecologist (including specialists in gynecological endocrinology and reproductive medicine, and gynecological oncology; LANR codes: $15,16,17)$, ophthalmologist (LANR code: 05), dermatologist (LANR code: 21), orthopedist (LANR code: 10), others (all specialist groups not mentioned above) and unknown. In this study, check-ups refer to visits for the supervision of pregnancy (EMB [German Uniform Evaluation Standard] code: 01770).

\section{Hospital Stays, Physical Therapy, and Sick Leave}

Hospital stays were identified by a hospital case identifier, with only fully inpatient hospital stays considered. The number and length of hospital stays were calculated.

Prescriptions for physical therapy are required for the service to be paid by health insurance in Germany. Use of physical therapy services were billed by position numbers where the first digit identifies the service provider, and the second and third digits identify the service type. The position numbers utilized in this study are listed in Table S3 (Supplementary Material). Position numbers for providers of outpatient treatments in accepted health resorts were not used by the study population, and therefore were not included in the analysis. The utilization of sick leave was calculated directly from the BARMER database.

\section{Pre-specified Comorbidities and Complications} Three pre-specified comorbidities were investigated in this study and were coded by ICD-10 codes. The three comorbidities investigated were: uveitis (H20.0, H20.1, H20.9), inflammatory bowel disease (K50.X, K51.X), and enthesitis (M72.2, M76.8, M76.9, M77.5, M77.8, M77.9), where $X$ refers to any number. Comorbidities had to be recorded as either an outpatient assured diagnosis or an inpatient diagnosis (hospital discharge diagnosis or secondary diagnosis) in order to be included in these analyses.

Complications during pregnancy, childbirth, and the postpartum period were defined by ICD-10 codes of O99.X (other maternal diseases classifiable elsewhere but complicating pregnancy, childbirth and the puerperium), where $X$ refers to any number.

\section{Pharmacological Treatments}

New prescriptions during pregnancy were defined as the first prescription of a drug during pregnancy with no prescription in the 180 days before pregnancy. Prescriptions were identified by a prescription identifier and the drug prescribed was identified by the Anatomical Therapeutic Chemical (ATC) code (Supplementary Material: Table S4). The prescription of any drug is reported, and this is also broken down into the following pre-specified categories: 'anti-inflammatory drugs' (defined by ATC code M01A [anti-inflammatory/anti-rheumatic products and non-steroids]), 'disease-specific drugs' (defined by ATC code M01C and additional codes for methotrexate; including the following therapies only: quinolines, gold preparations, penicillamine and similar agents, sulfasalazine and methotrexate), 'tumor necrosis factor inhibitor (TNFi) treatments' and 'other biologics 
and small molecules'. The specific drugs in each category are presented in Table S4 (Supplementary Material). The prescription of corticosteroids over the course of time was also investigated (Supplementary Material: Table S4).

\section{Hospitalization/New Prescription of Corticosteroids or Biologics}

The utilization of the above resources, as indicators of disease exacerbation, were defined in the following manner: (1) hospitalization due to the target indication, or (2) prescription of corticosteroids or biologics for the first time (i.e., there was no prescription of these drugs in the 180 days before the time period of interest; Supplementary Material: Table S4). Hospitalization/new prescription of corticosteroids or biologics was investigated in the following time periods of pregnancy: 180 days before pregnancy, during pregnancy, and 180 days after delivery. Women who were identified as utilizing these resources during more than one of these time periods were included in the analysis for each respective period.

\section{Analysis}

As a non-interventional retrospective study, the analyses carried out here are purely descriptive. No formal statistical testing was performed, so any differences reported are only numerically different and should be interpreted with caution. The results of the analyses in all groups have been extrapolated to the German statutory health insurance population, using age groupspecific weighting factors. Extrapolated values are reported rather than the numbers of patients identified from the database.

Utilization of check-ups, prescription of physical therapy or drugs, pre-specified comorbidities, pre-specified complications, preterm delivery, physician contact, specialty of physicians prescribing drugs, and hospitalization/ new prescription of corticosteroids or biologics are all reported as proportions of women. Utilization of sick leave was reported as the proportion of women who took any sick leave over the reported period. Age, number of check- ups, number of hospital stays, and length of hospital stays are all reported as the mean and standard deviation (SD).

\section{RESULTS}

\section{Patient Characteristics and Healthcare Resource Utilization}

In total, 2702 women with the IRDs of interest (axSpA: 1063; PsA: 660; RA: 979) and 6527 women with PSO in the BARMER database were identified as being pregnant during the study period. Pregnant women with a diagnosis of IRD or PSO during pregnancy and the agematched control groups had similar characteristics and HCRU, except for physical therapy and the presence of pre-specified comorbidities (uveitis, inflammatory bowel disease or enthesitis; Table 1). Preterm delivery was more frequent in women with IRD compared with the IRD control group, and consistent between women with PSO and the PSO control group (Table 1).

As expected, substantially higher proportions of women with IRD were in contact with internal medicine and rheumatology specialists during pregnancy compared with women without IRD (Fig. 2a). In addition, GPs, ophthalmologists, orthopedists, and internal medicine specialists were also seen more often by pregnant women with IRD than the control group (Fig. 2a). During pregnancy, higher proportions of women with PSO were in contact with dermatologists and GPs compared with women without a PSO diagnosis (Fig. 2b).

\section{Prescription of Treatments During Pregnancy}

During pregnancy, high proportions of women in all groups were prescribed prescription drugs (Table 1). Generally, prescription drugs were prescribed to a higher proportion of women with IRD and PSO than women without these diseases (Table 1). Anti-inflammatory drugs were prescribed to a higher proportion of women with IRD than women in the IRD 
Table 1 Characteristics, HCRU, and treatment of women with IRD or PSO, and the respective control groups, during pregnancy

\begin{tabular}{|c|c|c|c|c|}
\hline & \multicolumn{2}{|l|}{ IRD } & \multicolumn{2}{|l|}{ PSO } \\
\hline & $\begin{array}{l}\text { Women with IRD } \\
(n=2702)\end{array}$ & $\begin{array}{l}\text { IRD control group } \\
(n=2702)\end{array}$ & $\begin{array}{l}\text { Women with PSO } \\
(n=6527)\end{array}$ & $\begin{array}{l}\text { PSO control group } \\
(n=6527)\end{array}$ \\
\hline \multicolumn{5}{|l|}{ Characteristics and demographics } \\
\hline Age, mean (SD) & $32(4.3)$ & $32(4.3)$ & $31(5.0)$ & $31(5.0)$ \\
\hline Any pre-specified comorbidity ${ }^{\mathrm{b}}, n(\%)$ & $218(8.1)$ & $54(2.0)$ & $253(3.9)$ & $153(2.4)$ \\
\hline Any pre-specified complications ${ }^{c}, n(\%)$ & $963(35.7)$ & $934(34.6)$ & $2438(37.4)$ & $2164(33.2)$ \\
\hline Preterm delivery, $n$ (\%) & $191(7.1)$ & $145(5.4)$ & $352(5.4)$ & $335(5.1)$ \\
\hline \multicolumn{5}{|l|}{$H C R U$} \\
\hline Utilization of check-ups, $n(\%)$ & $2675(99.0)$ & $2666(98.6)$ & $6464(99.0)$ & $6436(98.6)$ \\
\hline Number of check-ups ${ }^{\mathrm{d}}$, mean $(\mathrm{SD})$ & $26(7.5)$ & $25(7.5)$ & $26(7.4)$ & $25(7.2)$ \\
\hline Number of hospital stays ${ }^{\mathrm{e}}$, mean (SD) & $1(0.7)$ & $1(0.7)$ & $1(0.7)$ & $1(0.6)$ \\
\hline Length of hospital stay, days, mean (SD) & $2(3.7)$ & $2(5.0)$ & $2(4.3)$ & $2(3.8)$ \\
\hline Physical therapy, $n$ (\%) & $792(29.3)$ & $299(11.1)$ & $1228(18.8)$ & $725(11.1)$ \\
\hline Sick leave, $n(\%)$ & $1560(57.7)$ & $1386(51.3)$ & $3957(60.6)$ & $3322(50.9)$ \\
\hline \multicolumn{5}{|l|}{ Pharmacological treatments ${ }^{\mathrm{f}}$} \\
\hline Any prescription drugg, $n(\%)$ & $2330(86.2)$ & $2143(79.3)$ & $5383(82.5)$ & $4975(76.2)$ \\
\hline Anti-inflammatory drugs ${ }^{\mathrm{h}}, n(\%)$ & $381(14.1)$ & $98(3.7)$ & $272(4.2)$ & $234(3.6)$ \\
\hline Disease-specific drugs, $n(\%)$ & $243(9.0)$ & $0(0.0)$ & $18(0.3)$ & $0(0.0)$ \\
\hline TNFi treatments, $n(\%)$ & $209(7.7)$ & $0(0.0)$ & $18(0.3)$ & $9(0.1)$ \\
\hline Other biologics, $n(\%)$ & $27(1.0)$ & $0(0.0)$ & $0(0.0)$ & $0(0.0)$ \\
\hline
\end{tabular}

Multiple answers were possible, and all percentages report the proportion of women unless otherwise stated. Utilization of sick leave was reported as the proportion of women who took any sick leave over the reported period

$H C R U$ healthcare resource utilization, IRD inflammatory rheumatic diseases, PSO psoriasis, SD standard deviation, TNFi tumor necrosis factor inhibitor

a Pregnant women of the same age and timing of pregnancy without a documented diagnosis

b Pre-specified comorbidities were uveitis, inflammatory bowel disease, and enthesitis

c Pre-specified complications during pregnancy, childbirth, and the postpartum period were defined by ICD-10 codes of O99.X (other maternal diseases classifiable elsewhere but complicating pregnancy, childbirth, and the puerperium), where $X$ refers to any number

${ }^{\mathrm{d}}$ Number of check-ups per person

e Number of hospital stays includes hospital stays for delivery

${ }^{f}$ The proportion of women receiving at least one prescription for a pharmacological treatment in each group, the pharmacological treatments in each group are defined in Supplementary Material: Table S4

g Any prescription drug includes drugs beyond those included in the pre-specified categories defined in Supplementary Material: Table S4

h Anti-inflammatory/anti-rheumatic products and non-steroids (defined by ATC code: M01A) 


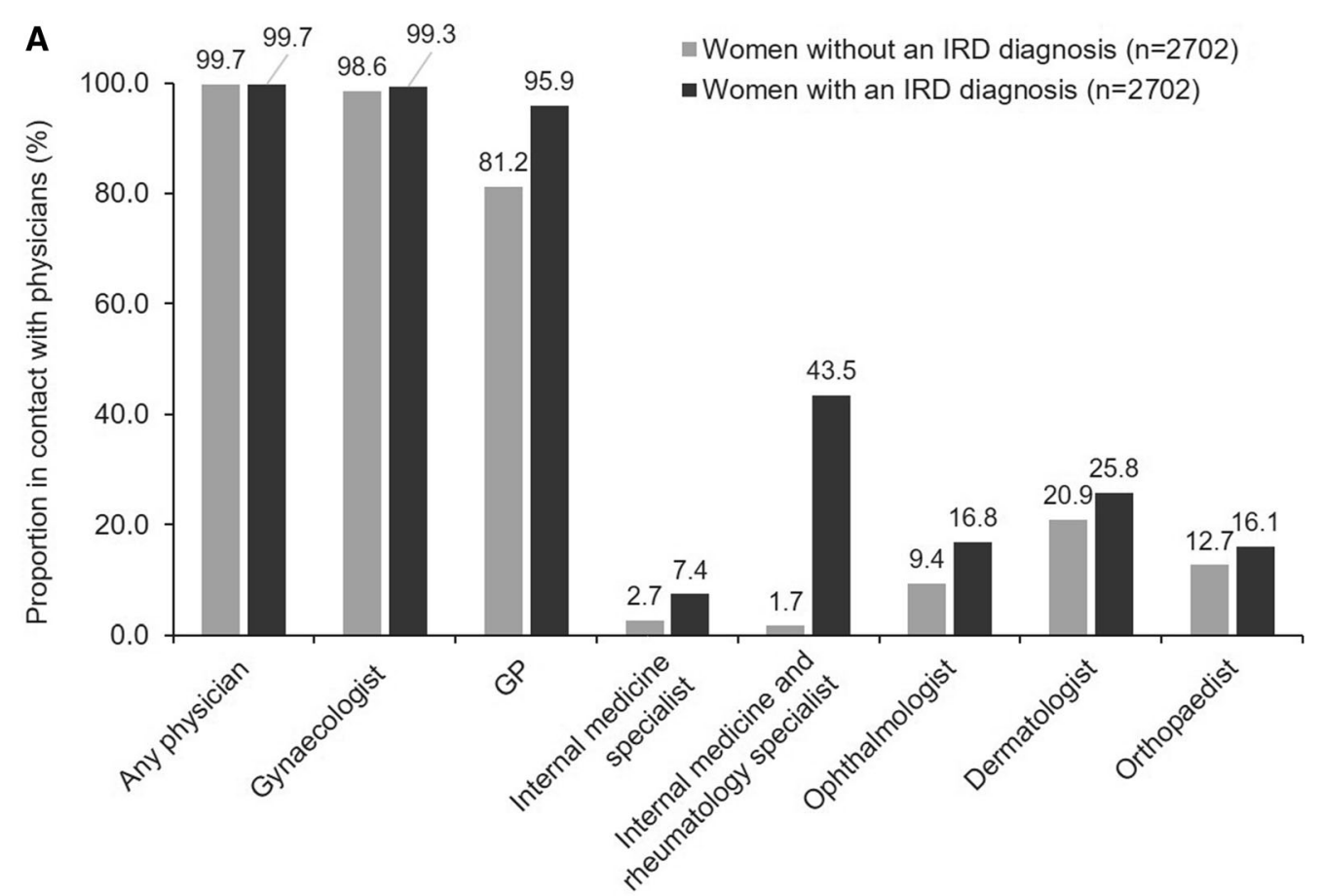

Physician group

B

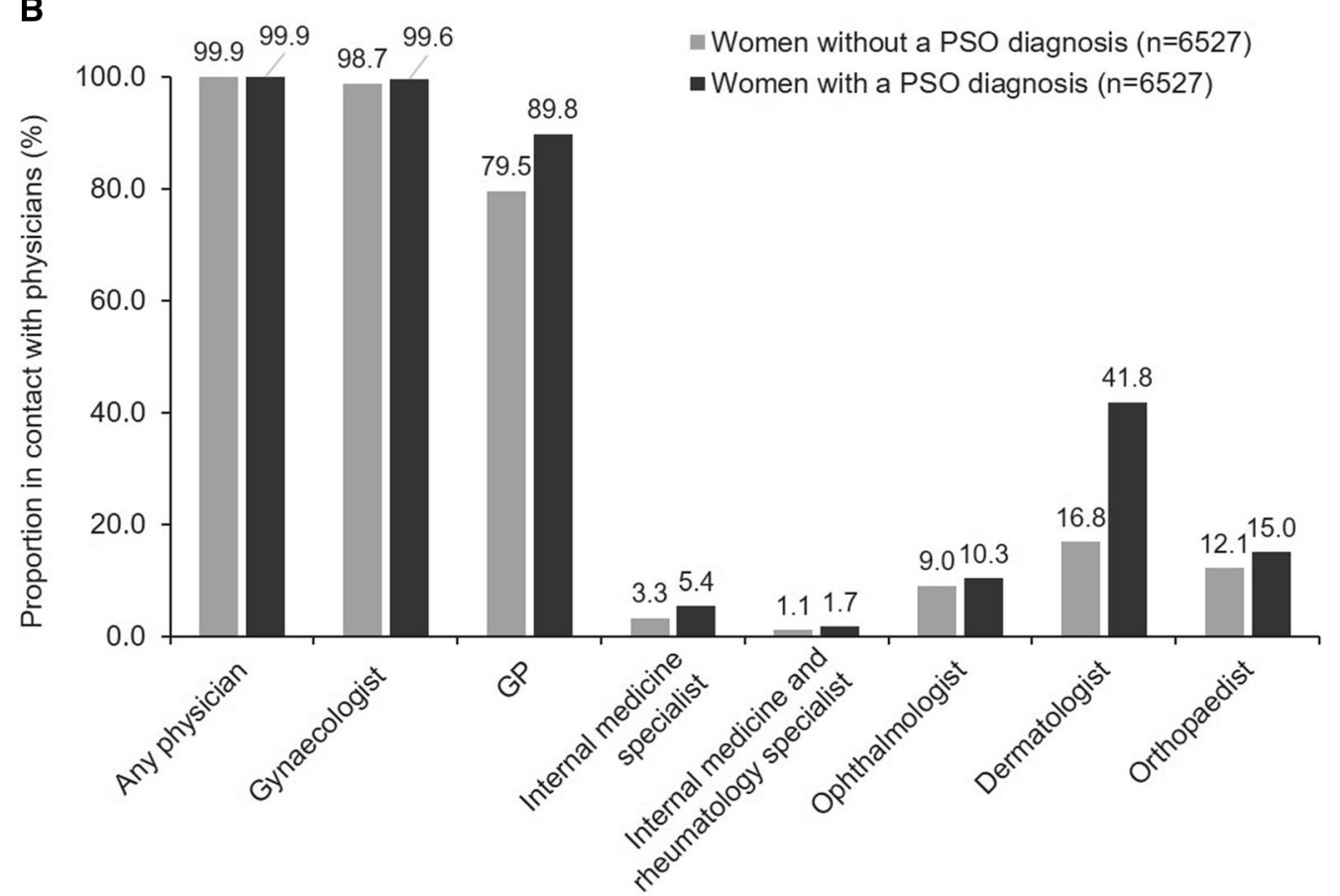

Physician group

Fig. 2 Specialty of physicians in contact with women with IRD or PSO, and the respective control groups, during pregnancy. a IRD. b PSO. Multiple answers were possible.
$G P$ general practitioner, IRD inflammatory rheumatic disease, PSO psoriasis 
control group (Table 1). Disease-specific drugs, TNFi treatments, and other biologics were prescribed to $9.0 \%, 7.7 \%$, and $1.0 \%$ of women with IRD during pregnancy, respectively (Table 1). Low proportions of women with PSO received prescriptions for disease-specific drugs, TNFi treatments, and other biologics during pregnancy (Table 1).

When assessing the specialty of physicians prescribing any drugs, pregnant women with IRD were more commonly prescribed drugs by GPs, internal medicine, and rheumatology specialists and dermatologists compared with women without IRD (Fig. 3a). Similarly, a greater proportion of women with PSO received prescriptions from GPs and dermatologists compared with women without PSO (Fig. 3c). Fewer women with IRD and PSO received new prescriptions from gynecologists during pregnancy, and to a lesser extent from GPs, when compared with women without these diagnoses (Fig. 3b, d).

The specialty of physicians prescribing disease-specific drugs and biologics were only investigated in pregnant women with IRD or PSO during pregnancy. Among women with IRD, the prescription of current or new diseasespecific drugs were most commonly provided by internal medicine and rheumatology specialists and GPs (Table 2). Ongoing prescriptions of biologics were most commonly prescribed to women with IRD by internal medicine and rheumatology specialists during pregnancy (Table 2). The proportion of women with IRD who were newly prescribed biologics during pregnancy was low (1\%). Among women with PSO, disease-specific drugs were prescribed by GPs $(0.1 \%$ of women) and internal medicine and rheumatology specialists ( $0.1 \%$ of women). Due to the low prescription rate of diseasespecific drugs and biologics to pregnant women with PSO ( $n<10$ per physician group), further data are not presented here.

\section{Treatment Patterns Over Time}

The pattern of prescriptions before pregnancy, during pregnancy, and after delivery was studied in pregnant women with IRD and PSO
(Fig. 4). The breakdown of the prescription patterns for axSpA, PsA, and RA over time are also shown (Supplementary Material: Figure S1). Before pregnancy, prescriptions for diseasespecific drugs were received by $15.0 \%$ of women with IRD and during pregnancy this decreased to $9.0 \%$ of women with IRD. After delivery, the proportion of women with IRD with prescriptions for disease-specific drugs remained relatively stable at $9.7 \%$ (Fig. 4). The proportion of women with PSO receiving prescriptions for disease-specific drugs was low over the course of pregnancy (0.1-0.6\%; Fig. 4).

TNFi treatments were prescribed to $13.1 \%$ of women with IRD before pregnancy, which decreased to $7.7 \%$ of women during pregnancy. After delivery, the proportion of women with IRD prescribed TNFi treatments increased slightly to $9.7 \%$, but this did not reach prepregnancy levels (Fig. 4a). Notably, the decrease in the prescription of TNFi treatments after delivery was most pronounced in women with PsA, whereas in women with axSpA the prescription rate returned to pre-pregnancy levels (Supplementary Material: Figure S1). Adalimumab and etanercept were the most common TNFi treatments prescribed to women with IRD before and during pregnancy. After delivery, adalimumab and certolizumab pegol were the most common TNFi treatments prescribed to women with IRD (patient numbers are low for most individual TNFi treatments; Fig. 4a). In contrast, the proportion of women with PSO receiving any TNFi treatment prescriptions remained stable at a low level before, during, and after pregnancy (0.3-0.4\%; Fig. 4b).

Other biologic therapies were prescribed to $2.7 \%$ of women with IRD and $0.6 \%$ of women with PSO before pregnancy. This proportion declined during pregnancy but increased back to pre-pregnancy levels after delivery (Fig. 4). Secukinumab and ustekinumab were not prescribed during pregnancy in women with IRD or PSO (Fig. 4).

In the 180 days before pregnancy, corticosteroids were prescribed to $24.4 \%$ of women with IRD and $3.2 \%$ of women with PSO. During pregnancy these proportions decreased slightly to $20.1 \%$ of women with IRD and $2.1 \%$ of women with PSO, and then remained 


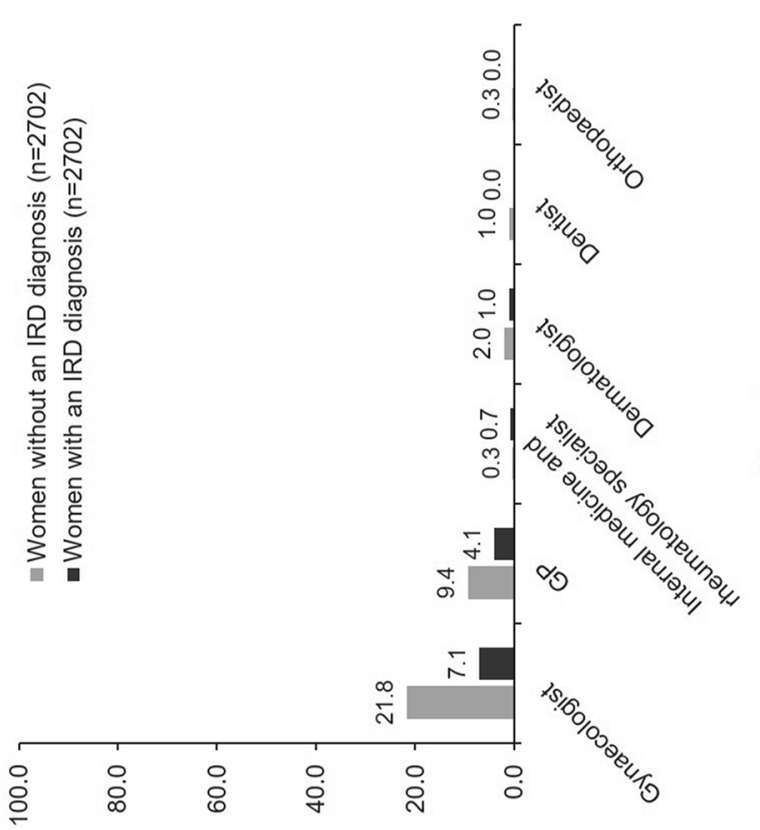

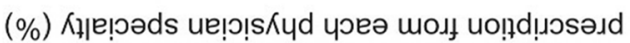

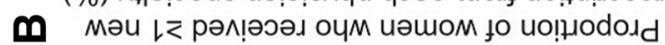

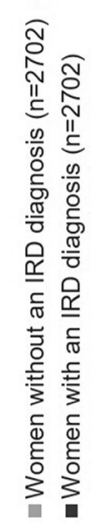

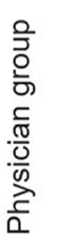

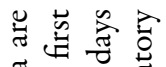

今ึ

ஓं ம.

$\circ-\exists$.

䒕 $\cong$

के

更

吾苔

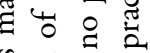

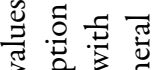

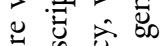

选造芯

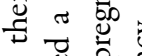

苛

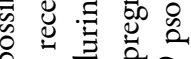

on $y$

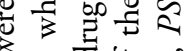

菏㟧

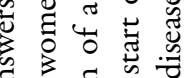

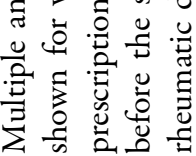

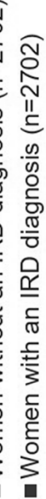

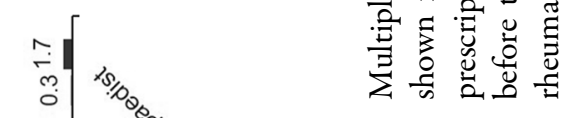

完总骂

पे ठำ

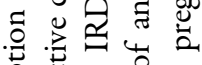

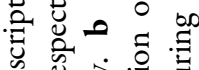

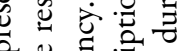

픔

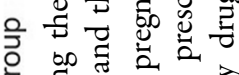

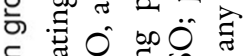

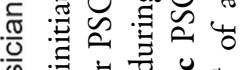

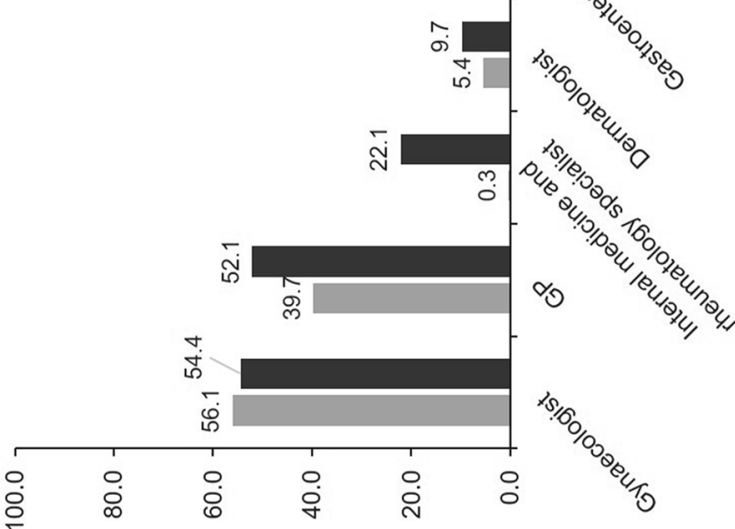

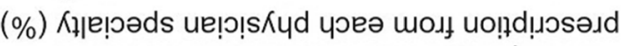
l< рәл!әәәд очм иәшом jo uo!̣ododd ^ैة

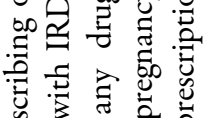

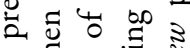

ป ฐี ฐ

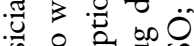

爷氙式

㟧䒕艺

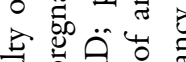

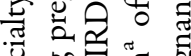

ฉั. $\approx$.

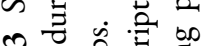

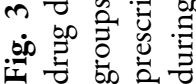




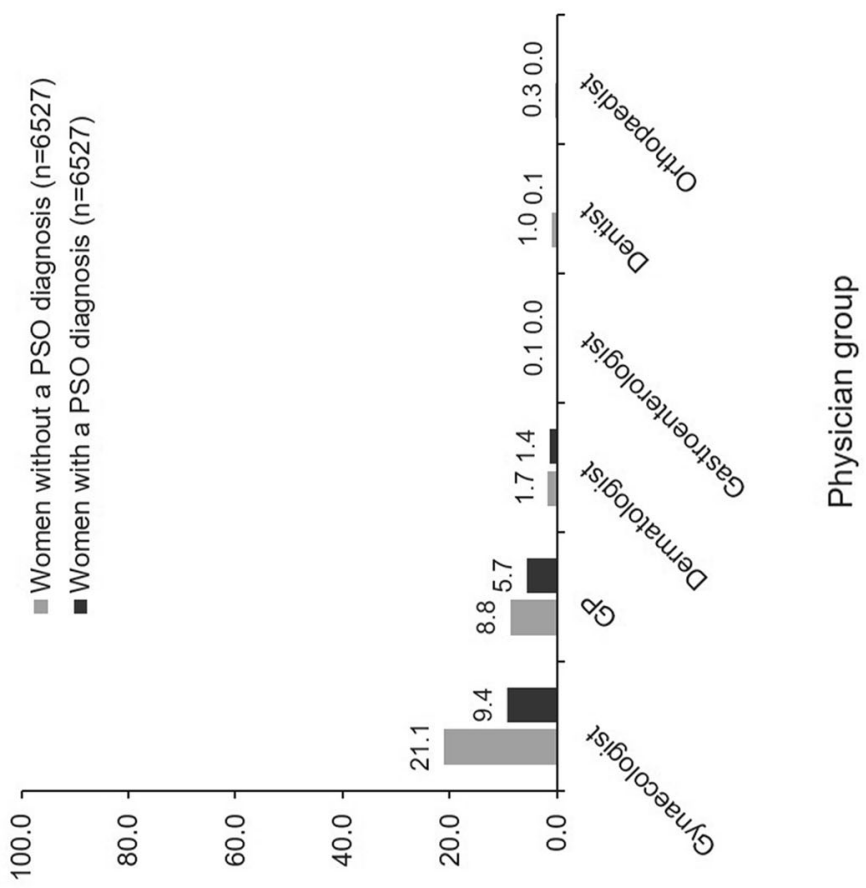

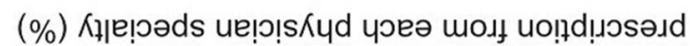

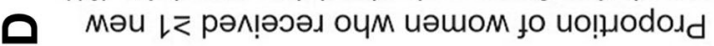

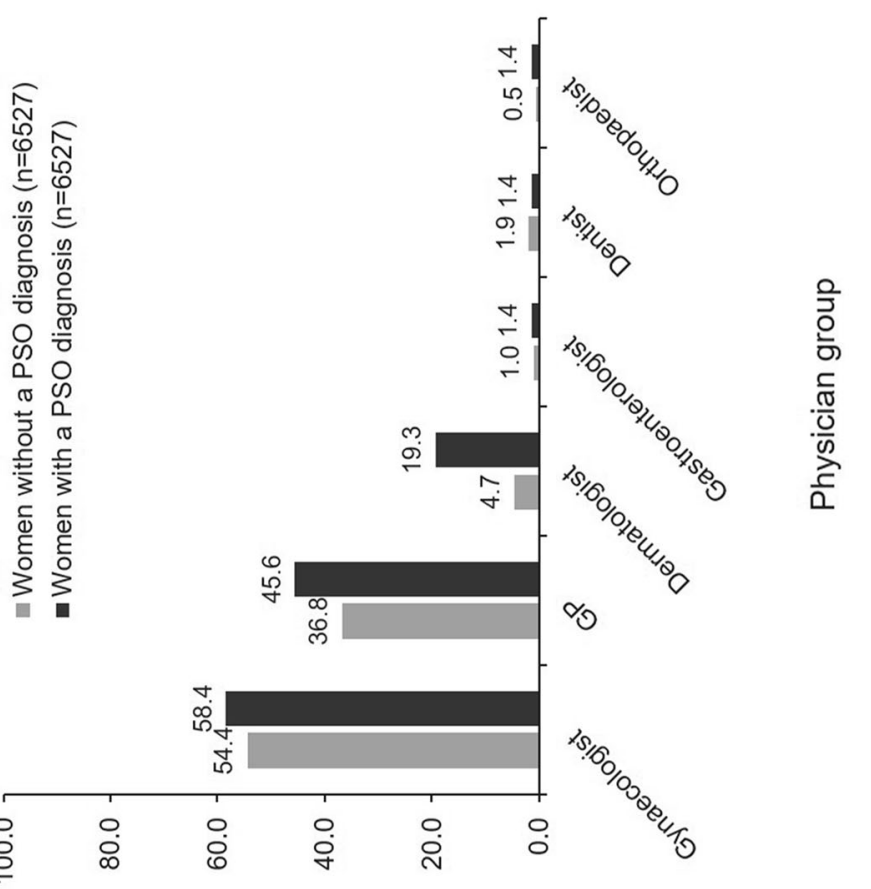

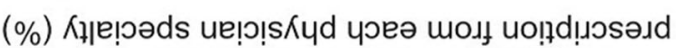

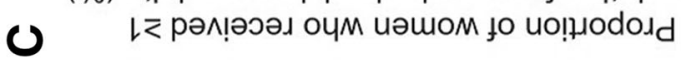


Table 2 Specialty of physicians prescribing or initiating the prescription of disease-specific drugs or biologics during pregnancy to women with IRD

\begin{tabular}{lc}
\hline & $\begin{array}{c}\text { Women with a } \\
\text { diagnosis of IRD } \\
(\boldsymbol{n}=\mathbf{2 7 0 2})\end{array}$ \\
\hline $\begin{array}{l}\text { Prescription of disease-specific drugs according to } \\
\text { physician specialty, } n \text { (\%) }\end{array}$ & $134(5.0)$ \\
Internal medicine and & $108(4.0)$ \\
rheumatology specialist & $9(0.3)$ \\
GP & $8(0.3)$ \\
Gastroenterologist & $18(0.7)$ \\
Dermatologist & $9(0.3)$ \\
New prescription ${ }^{\text {a }}$ of disease-specific drugs according to \\
physician specialty, $n$ (\%) \\
Internal medicine and \\
rheumatology specialist \\
GP
\end{tabular}

Multiple answers were possible. Data are shown for women who received a prescription of at least one drug. Due to the low prescription rate of disease-specific drugs and biologics to pregnant women with PSO this data is not presented here

$G P$ general practitioner, IRD inflammatory rheumatic diseases

a The first prescription of a drug during pregnancy, with no prescription in the 180 days before the start of the pregnancy stable after delivery (Fig. 4). Interestingly, the pattern of corticosteroid prescription over time was different between the three IRD diagnoses included in this study. The proportion of women with axSpA who were prescribed corticosteroids decreased sharply from $17.1 \%$ before pregnancy to $5.1 \%$ during pregnancy, and then remained stable after delivery (Supplementary Material: Figure S1a). In comparison, the proportion of women with PsA who were prescribed corticosteroids decreased slightly over time, while a small increase over time was observed among women with RA (Supplementary Material: Figure S1b and S1c).

\section{Hospitalizations or New Prescriptions of Corticosteroids or Biologics Over Time}

The proportion of women who required hospitalization or a new prescription of corticosteroids/biologics in the 180 days before pregnancy, during pregnancy, and in the 180 days after delivery was investigated and could potentially indicate disease exacerbation (Fig. 5). Before pregnancy, the proportion of women with IRD with at least one documented use of these specific resources was $9.0 \%$; this decreased to $5.1 \%$ during pregnancy. After delivery, the proportion of women with IRD who utilized these resources increased beyond pre-pregnancy levels to $11.1 \%$ (Fig. 5). Utilization of these resources occurred in a smaller proportion of women with PSO before, during and after pregnancy compared with women with IRD, but a similar reduction during pregnancy was observed (before: 3.5\% of women, during: $1.9 \%$ of women). The proportion of women with PSO utilizing these resources increased after delivery (2.7\%) but did not reach pre-pregnancy levels (Fig. 5).

\section{DISCUSSION}

This retrospective claims database analysis of German sickness fund data investigated the HCRU and treatment patterns of pregnant women with IRD and PSO in Germany. The data presented here show that for women with IRD there is a reduction in treatment during 


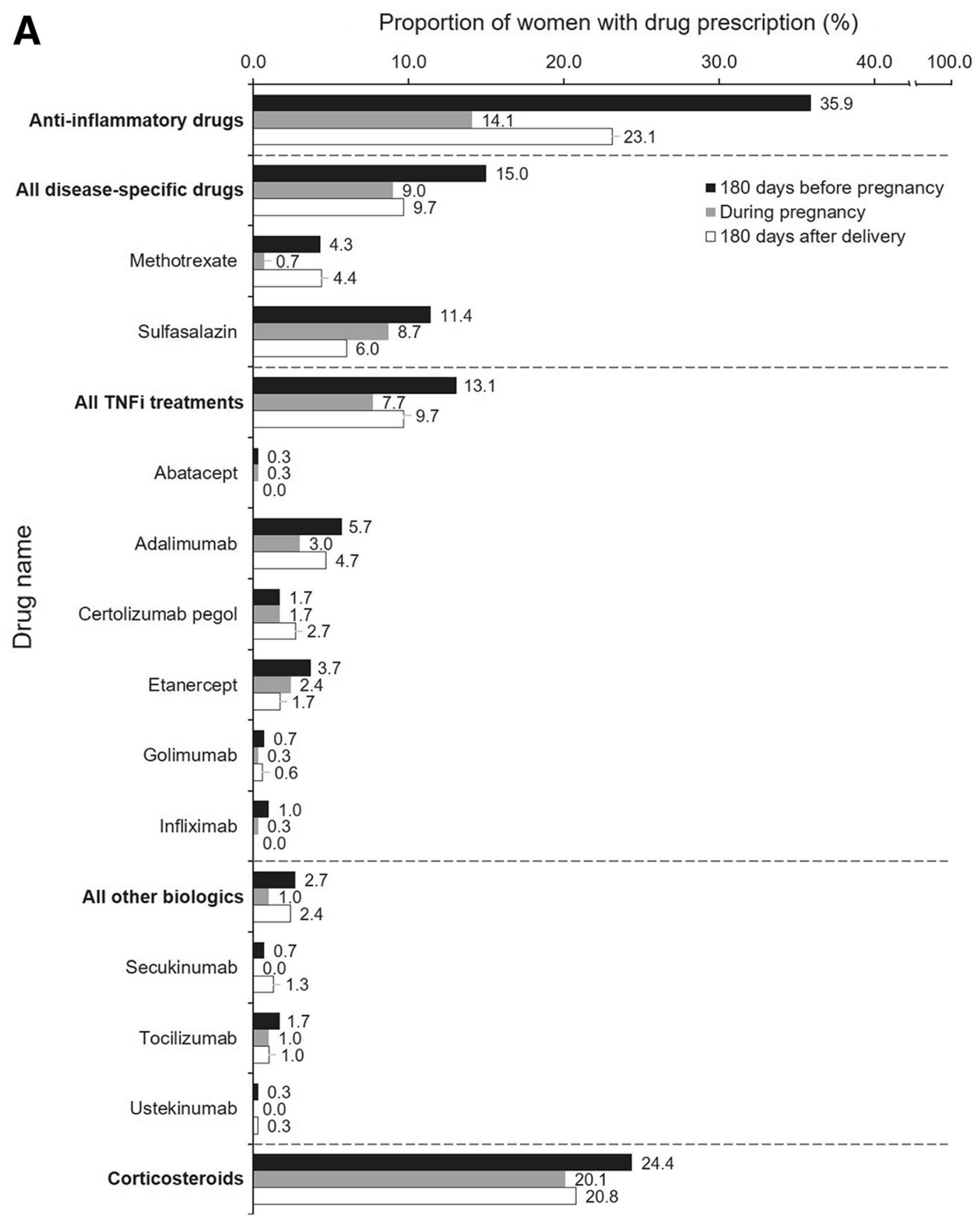

Fig. 4 Proportion of women with IRD or PSO who were prescribed disease-specific drugs, biologics and corticosteroids over time. a IRD $(n=2702)$. b PSO $(n=6527)$. Multiple answers were possible. Data are shown for women

pregnancy and that many women did not return to their pre-pregnancy treatments in the 180 days after delivery, despite potential signs of disease exacerbation in this period. who received a prescription of at least one drug. IRD inflammatory rheumatic diseases, PSO psoriasis, TNFi tumor necrosis factor inhibitor

The EULAR points to consider, published in 2016 [19], and ACR guidelines, published in 2020 [6], provide up-to-date guidance on the treatment of pregnant women with chronic 


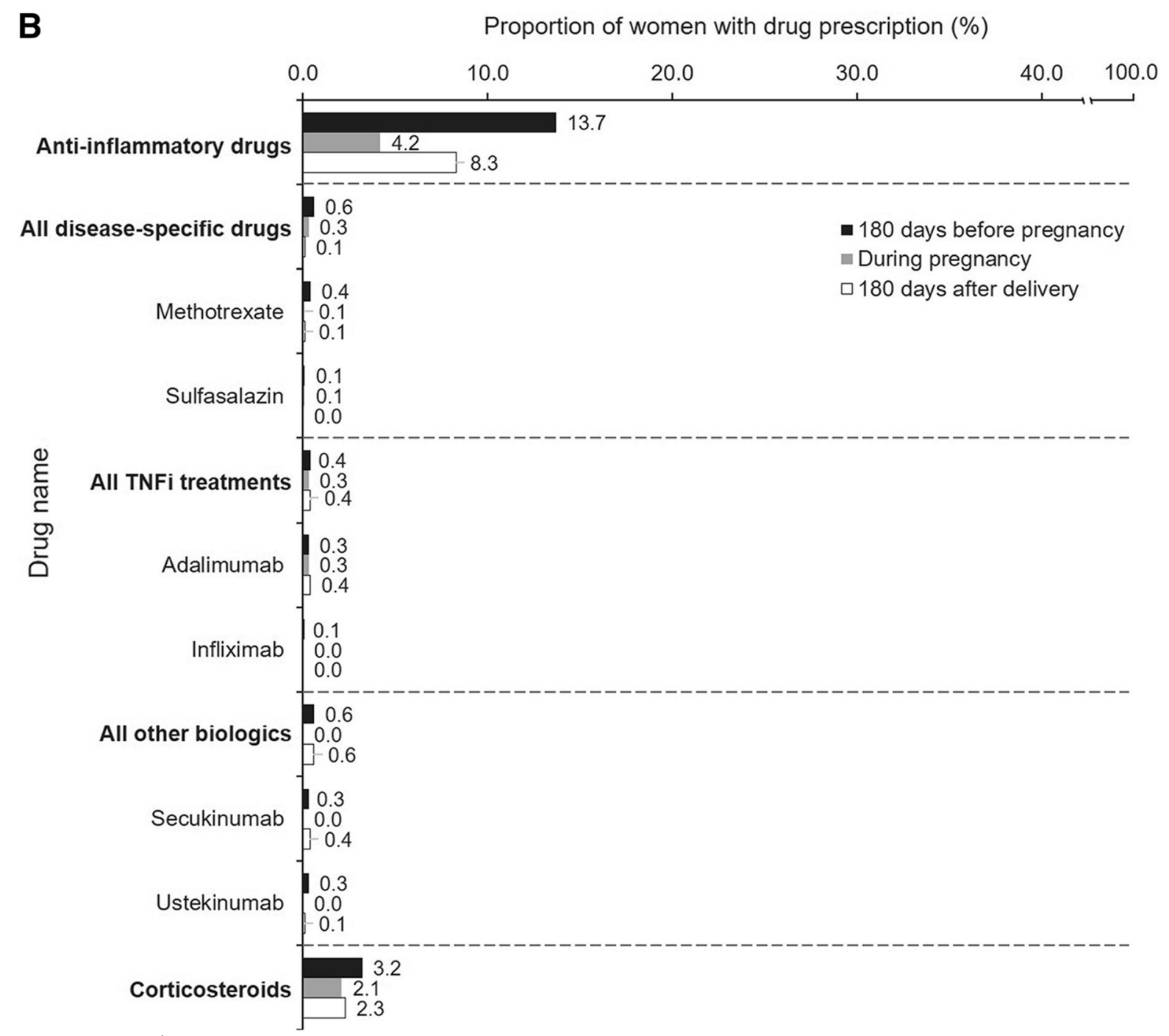

Fig. 4 continued

rheumatic diseases and indicate that the effective management of these conditions during pregnancy is possible. Claims database analyses, such as those presented here, can confirm the relevance of such recommendations, and may help to inform future versions and encourage adherence to these recommendations.

In this study, prescriptions for disease-specific drugs, TNFi treatments and other biologics decreased during pregnancy for women with IRD diagnoses. This is in line with a previous cohort study of HCRU data in the US that showed a reduction in the proportion of women with RA receiving biologic disease-modifying anti-rheumatic drug (DMARD) prescriptions, and some non-biologic DMARD prescriptions, from the first to the third trimester of pregnancy [27]. Another US cohort study also showed a reduction in the prescription of immunomodulatory agents (including steroids, non-biologic DMARDs, and biologic DMARDs [bDMARDs]) to women with AS, PsA, and RA during pregnancy [28]. Furthermore, a Canadian observational cohort study showed that $38 \%$ of women with a range of auto-immune diseases, including RA, discontinued biologic treatment by the second trimester of pregnancy [29]. Together, these studies all support the trend that treatment is often reduced during pregnancy in women with IRD. 


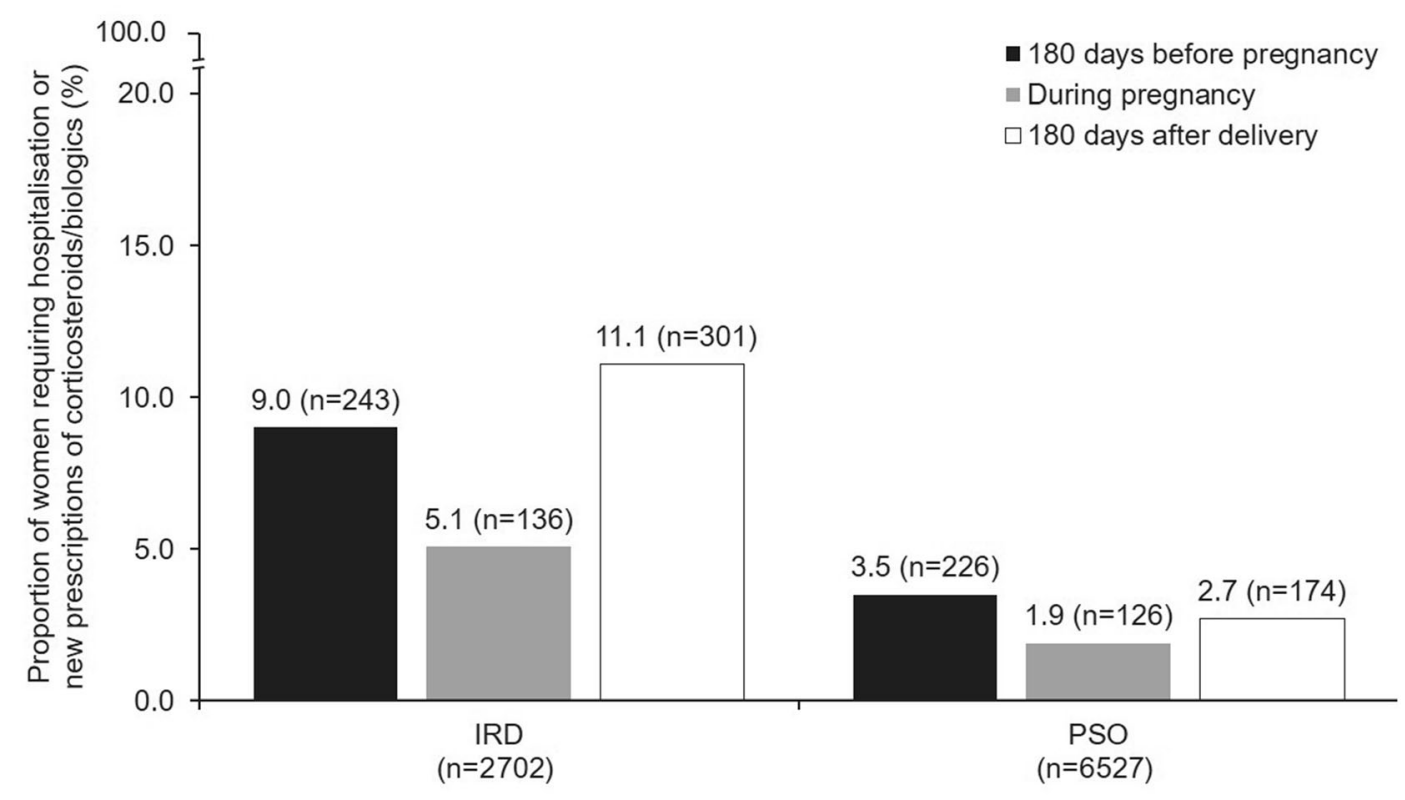

Fig. 5 Proportion of pregnant women with IRD or PSO and documentation of hospitalization or new prescriptions of corticosteroids/biologics over time. IRD inflammatory rheumatic diseases, PSO psoriasis

Prescriptions for corticosteroids in women with IRD decreased slightly during pregnancy and then remained stable after delivery in this study. However, the pattern observed was different for patients with axSpA, PsA, and RA, with women with axSpA showing the most substantial decrease in corticosteroid prescription during pregnancy. It was observed that many women with IRD, and in particular those with RA and PsA, received corticosteroid prescriptions during pregnancy. This is in line with previous reports in the literature for the corticosteroid prednisolone and with the EULAR points to consider, which support the use of prednisolone during pregnancy to control active disease $[19,30]$.

The proportion of women with PSO who received disease-specific drugs, TNFi treatment, and other biologic prescriptions was low $(<1 \%)$ in this study and therefore clear conclusions cannot be drawn. However, only $3 / 6$ TNFi treatments and $4 / 10$ other biologics investigated in this study were approved by the European Medicines Agency (EMA) for use in the treatment of PSO in the European Union (EU) during the study period. The PSO-MOTHER retrospective cohort study in Italy reported a reduction in systemic drug prescriptions in patients with PSO during pregnancy and an increase in the 91 days after delivery, yet the levels after delivery did not reach pre-pregnancy prescription rates [31]. The PSO-MOTHER study also showed that prescriptions for biologics decreased during pregnancy and then increased after delivery [31]. Reasons suggested by the PSO-MOTHER study for the reduction in prescriptions during pregnancy center around concerns by mothers over the impact of treatment on pregnancy outcomes [31].

The overall reduction in treatment for women with IRD during pregnancy observed in this study, including the reduction in new prescriptions by GPs and gynecologists and the low level of prescriptions by internal medicine and rheumatology specialists, is likely linked to concerns over the impact of treatment on pregnancy outcomes. This is supported by a recent survey in women of childbearing age with axSpA, PsA, and RA, which reported that stopping treatment during pregnancy was driven by the fear of harming the fetus in $78 \%$ of women [32]. The observed lack of return to treatment after delivery in this study could be linked to concerns over the impact of treatment on breastfeeding. This is also supported by the above-mentioned survey in which $66 \%$ of 
mothers felt that they had to make a decision between receiving treatment and breastfeeding [32].

The treatment patterns observed in this study largely align with the EULAR points to consider published in 2016 [19]. For example, methotrexate has been recommended to be withdrawn before pregnancy in patients with rheumatic diseases [19], and only $0.7 \%$ of women with IRDs were prescribed methotrexate during pregnancy in this study. The small proportion of women receiving methotrexate during pregnancy $(0.7 \%)$ could be attributed to these prescriptions being issued before the pregnancy became known. This is supported by a previous study that suggested that half of pregnancies in women of childbearing age with chronic rheumatic disease are not actively planned [32]. Furthermore, the TNFi treatments adalimumab and etanercept have been recommended for use during the first part of pregnancy (until gestation week 20 and 30-32, respectively) and breastfeeding, and the TNFi treatment certolizumab pegol has been recommended for use throughout pregnancy and breastfeeding [19]. We showed here that women with IRD received prescriptions for these drugs both during pregnancy and after delivery.

Overall, treatment options should be carefully considered to ensure that the most appropriate treatment is prescribed before, during and after pregnancy, as while some treatment options are high risk, there are more suitable treatment options available [19, 33]. Country-specific resources such as Embryotox [34], which provides independent information on the tolerance of a range of medications during pregnancy and breastfeeding, can be a valuable resource for physicians when treating pregnant women. Despite this, there is a need for further investigations into the safety of some treatments during pregnancy to ensure women can confidently continue their treatments [19].

Hospitalizations and new prescriptions of corticosteroids/biologics may indicate potential disease exacerbation or worsening. The utilization of these resources was reported in a smaller proportion of women with IRD and PSO during pregnancy compared with the corresponding 180 days before pregnancy and after delivery.
After pregnancy, the proportion of women utilizing these resources increased beyond the prepregnancy level in women with IRD, and almost back to the pre-pregnancy level in women with PSO. These observations are in line with previous studies, which have shown improvements in disease activity during pregnancy and deterioration postpartum in women diagnosed with RA, PsA, and PSO [11-15]. Changes in autoimmune disease activity during and after pregnancy have previously been linked to changes in hormones and cytokines during these periods [35]. The reduction in treatment over pregnancy and the postpartum period observed in this study could also contribute to the increased frequency of disease flares after delivery.

The preterm delivery rates observed in pregnant women with and without IRD and PSO in this study were relatively consistent (5.1-7.1\%) and are in line with published estimates of the preterm delivery rate in Europe in 2014 (8.7\%; uncertainty interval 6.3-13.3\%) [36].

\section{Study Limitations}

As with all database studies, this study was limited by the potential for misdiagnoses and miscoding, which may impact the study conclusions. As a non-interventional retrospective study, the analyses carried out here are purely descriptive and, as a result, conclusions cannot be drawn regarding associations. Furthermore, this database study did not have access to clinical data regarding disease severity across the patient populations, which is included in some US claims databases, and therefore conclusions cannot be drawn on whether the treatment patterns observed were sufficient. Drug prescriptions in this study were not assigned to specific diseases, therefore it cannot be ruled out that drug prescriptions were due to conditions other than IRD or PSO.

Here, we have attempted to approximate disease exacerbation using the proportion of women requiring hospitalization or the initiation of corticosteroids or biologic prescriptions. However, disease exacerbations which were not medically treated would not have been detected in this analysis, so these results only provide an 
indication of disease worsening. Notably, detecting disease exacerbations using claims data that do not include data on disease activity is inherently difficult. In order to close this research gap, pregnancy registries have been established in several European countries, enabling more reliable analysis of disease course over the pregnancy period $[37,38]$.

In this study, the ICD-10 code O09, which categorizes the length of pregnancy into weekly periods, was used to calculate the length and start of pregnancy. The ICD-10 code O09 has a range of 3-9 weeks in each category which generally gets shorter as the length of pregnancy increases. Here, the last-mentioned week of pregnancy was used for each category (maximum 42 weeks) to calculate the length of pregnancy. It must be noted that this approach creates a risk of misclassification of the length of pregnancy and a possible bias in separating the periods before and during pregnancy and after delivery in women with shorter pregnancy durations. As described in the results, the proportion of women with PSO and IRD with preterm birth in this study ranged from 5.4 to $7.1 \%$. Therefore, in the majority of pregnant women with IRD and PSO, the pregnancy lasted longer than 36 weeks so was not classified as pre-term birth [39], and thus fell within the category of "37-41 completed weeks" (ICD O09.6) or "> 41 completed weeks" (ICD O09.7). In an American prospective cohort study that examined 125 women with singleton live births and used the last menstrual period to determine gestational length, the average length of pregnancy was 40-41 weeks [40]. Together, this suggests that, based on the definition used here, the risk of misclassification of the length of pregnancy in the present study was low.

Furthermore, the inclusion of only women with a full-term delivery in this study could introduce immortal time bias, as women who did not carry to term because of their IRD or PSO diagnosis would be excluded from the study. In addition, the exact date when the pregnancy became known and whether women were breastfeeding were not captured in the study, but both of these factors could impact the treatments received by women. The study also did not assess the timing of prescriptions or hospitalization/new prescription of corticosteroids or biologics during pregnancy and therefore details about which stage of pregnancy these events occurred during is not covered. Further information could also be gained from looking at a longer period of time after delivery when women are less likely to still be breastfeeding.

Despite the limitations discussed here, retrospective database studies are a useful approach by which disease and treatment patterns can be studied in patients in real-life clinical practice. The longitudinal nature of the data in claims databases allow the study of healthcare data over a longer period, with no recall or non-responder bias. Furthermore, as all insured persons are included in claims databases, regardless of their health states, even hard-to-reach groups are included in these studies.

\section{CONCLUSIONS}

In conclusion, this analysis provides key information on the treatment patterns of pregnant women with IRD and PSO in Germany. Most importantly, this study showed that large proportions of women with IRD did not return to their pre-pregnancy treatments in the 180 days after delivery, despite signs of disease exacerbation in this period, approximated by hospitalization and the initiation of treatment with corticosteroids/biologics. The identification of this unmet need in these women highlights that postpartum care should be prioritized for women with IRD and this could improve patient outcomes.

\section{ACKNOWLEDGEMENTS}

The authors acknowledge Susana Lobo Berastegui, MSc, MBA, and Frederik Fierens, MSc, PhD, UCB Pharma, Brussels, Belgium, for publication coordination.

Funding. This study and the Rapid Service Fee was funded by UCB Pharma. 
Medical Writing. The authors acknowledge Beverley Wilson, PhD, and Sarah Jayne Clements, PhD, Costello Medical, UK, for medical writing and editorial assistance based on the authors' input and direction. This was funded by UCB Pharma in accordance with Good Publication Practice (GPP3) guidelines (http:// www.ismpp.org/gpp3).

Authorship. All named authors meet the International Committee of Medical Journal Editors (ICMJE) criteria for authorship for this article, take responsibility for the integrity of the work as a whole, and have given their approval for this version to be published.

Authorship Contributions. Substantial contributions to study conception and design: Katja Blaschke, Ursula Marschall, Wojciech Dombrowsky, Lars Joeres, Ingrid Schubert; substantial contributions to the analysis: Katja Blaschke, Ursula Marschall, Ingrid Schubert; substantial contributions to the interpretation of the data: Katja Blaschke, Rebecca FischerBetz, Ursula Marschall, Wojciech Dombrowsky, Lars Joeres, Tanja Heidbrede, Ingrid Schubert; drafting the article or revising it critically for important intellectual content: Katja Blaschke, Rebecca Fischer-Betz, Ursula Marschall, Wojciech Dombrowsky, Lars Joeres, Tanja Heidbrede, Ingrid Schubert; final approval of the version of the article to be published: Katja Blaschke, Rebecca Fischer-Betz, Ursula Marschall, Wojciech Dombrowsky, Lars Joeres, Tanja Heidbrede, Ingrid Schubert.

Disclosures. Katja Blaschke, Ingrid Schubert: PMV has received research funds from UCB Pharma to conduct the study; Rebecca FischerBetz: Received speaking and consulting honoraria from UCB Pharma; Ursula Marschall: Employee of BARMER Statutory Health Insurance Fund; Wojciech Dombrowsky, Lars Joeres, Tanja Heidbrede: Employees of UCB Pharma.

Compliance with Ethics Guidelines. Ethical approval was not required for this study as the data analyzed were retrospective and were pseudonymized and anonymized, so that the subsequent assignment to individual patient data was impossible. Direct and project-specific access to the BARMER sickness fund W-DHW was contracted between BARMER and the PMV Research Group.

Data Availability. Data from non-clinical studies are outside of UCB Pharma's data sharing policy.

Prior Presentation. An abstract summarizing the key data from this manuscript has been submitted to, and subsequently accepted as an oral presentation for, the 11th International Conference on Reproduction, Pregnancy and Rheumatic Diseases (RheumaPreg 2021; Stresa, Lake Maggiore, Italy; 26-28 August 2021).

Open Access. This article is licensed under a Creative Commons Attribution-NonCommercial 4.0 International License, which permits any non-commercial use, sharing, adaptation, distribution and reproduction in any medium or format, as long as you give appropriate credit to the original author(s) and the source, provide a link to the Creative Commons licence, and indicate if changes were made. The images or other third party material in this article are included in the article's Creative Commons licence, unless indicated otherwise in a credit line to the material. If material is not included in the article's Creative Commons licence and your intended use is not permitted by statutory regulation or exceeds the permitted use, you will need to obtain permission directly from the copyright holder. To view a copy of this licence, visit http://creativecommons.org/licenses/by$\mathrm{nc} / 4.0 /$.

\section{REFERENCES}

1. Salaffi F, Di Carlo M, Carotti M, Farah S, Ciapetti A, Gutierrez M. The impact of different rheumatic diseases on health-related quality of life: a comparison with a selected sample of healthy individuals using SF-36 questionnaire, EQ-5D and SF-6D utility values. Acta Biomed. 2019;89:541-57.

2. Strand V, Singh JA. Patient burden of axial spondyloarthritis. J Clin Rheumatol. 2017;23: 383-91. 
3. Sarkar R, Chugh S, Bansal S. General measures and quality of life issues in psoriasis. Indian Dermatol Online J. 2016;7:481-8.

4. Mitchell K, Kaul M, Clowse ME. The management of rheumatic diseases in pregnancy. Scand J Rheumatol. 2010;39:99-108.

5. Vena GA, Cassano N, Bellia G, Colombo D. Psoriasis in pregnancy: challenges and solutions. Psoriasis (Auckl). 2015;5:83-95.

6. Sammaritano LR, Bermas BL, Chakravarty EE, et al. 2020 American college of rheumatology guideline for the management of reproductive health in rheumatic and musculoskeletal diseases. Arthritis Care Res (Hoboken). 2020;72:461-88.

7. Nørgaard M, Larsson H, Pedersen L, et al. Rheumatoid arthritis and birth outcomes: a Danish and Swedish nationwide prevalence study. J Intern Med. 2010;268:329-37.

8. Bharti B, Lee SJ, Lindsay SP, et al. Disease severity and pregnancy outcomes in women with rheumatoid arthritis: results from the organization of teratology information specialists autoimmune diseases in pregnancy project. J Rheumatol. 2015;42: 1376-82.

9. Zbinden A, van den Brandt S, Østensen M, Villiger PM, Förger F. Risk for adverse pregnancy outcome in axial spondyloarthritis and rheumatoid arthritis: disease activity matters. Rheumatology (Oxford). 2018;57:1235-42.

10. Bröms G, Haerskjold A, Granath F, Kieler H, Pedersen L, Berglind IA. Effect of maternal psoriasis on pregnancy and birth outcomes: a population-based cohort study from Denmark and Sweden. Acta Derm Venereol. 2018;98:728-34.

11. Berman M, Zisman D, Wollman J, et al. The effect of pregnancy on disease activity in patients with psoriatic arthritis. J Rheumatol. 2018;45:1651-5.

12. de Man YA, Dolhain RJ, van de Geijn FE, Willemsen SP, Hazes JM. Disease activity of rheumatoid arthritis during pregnancy: results from a nationwide prospective study. Arthritis Rheum. 2008;59: 1241-8.

13. Jethwa H, Lam S, Smith C, Giles I. Does rheumatoid arthritis really improve during pregnancy? A Systematic review and metaanalysis. J Rheumatol. 2019;46:245-50.

14. Murase JE, Chan KK, Garite TJ, Cooper DM, Weinstein GD. Hormonal effect on psoriasis in pregnancy and post partum. Arch Dermatol. 2005;141: 601-6.
15. Polachek A, Li S, Polachek IS, Chandran V, Gladman D. Psoriatic arthritis disease activity during pregnancy and the first-year postpartum. Semin Arthritis Rheum. 2017;46:740-5.

16. Østensen M, Fuhrer L, Mathieu R, Seitz M, Villiger PM. A prospective study of pregnant patients with rheumatoid arthritis and ankylosing spondylitis using validated clinical instruments. Ann Rheum Dis. 2004;63:1212-7.

17. Ursin K, Lydersen S, Skomsvoll JF, Wallenius M. Disease activity during and after pregnancy in women with axial spondyloarthritis: a prospective multicentre study. Rheumatology (Oxford). 2018;57:1064-71.

18. Mouyis M. Postnatal care of woman with rheumatic diseases. Adv Ther. 2020;37:3723-31.

19. Götestam Skorpen C, Hoeltzenbein M, Tincani A, et al. The EULAR points to consider for use of antirheumatic drugs before pregnancy, and during pregnancy and lactation. Ann Rheum Dis. 2016;75: 795-810.

20. Menter A, Gelfand JM, Connor C, et al. Joint American Academy of Dermatology-National Psoriasis Foundation guidelines of care for the management of psoriasis with systemic nonbiologic therapies. J Am Acad Dermatol. 2020;82:1445-86.

21. Menter A, Strober BE, Kaplan DH, et al. Joint AADNPF guidelines of care for the management and treatment of psoriasis with biologics. J Am Acad Dermatol. 2019;80:1029-72.

22. Nast A, Gisondi P, Ormerod AD, et al. European S3Guidelines on the systemic treatment of psoriasis vulgaris-Update 2015-Short version-EDF in cooperation with EADV and IPC. J Eur Acad Dermatol Venereol. 2015;29:2277-94.

23. Nast A, Spuls PI, van der Kraaij G, et al. European S3-Guideline on the systemic treatment of psoriasis vulgaris-Update Apremilast and SecukinumabEDF in cooperation with EADV and IPC. J Eur Acad Dermatol Venereol. 2017;31:1951-63.

24. Schneeweiss S, Avorn J. A review of uses of health care utilization databases for epidemiologic research on therapeutics. J Clin Epidemiol. 2005;58: 323-37.

25. Wiegand HF, Saam J, Marschall U, et al. Challenges in the transition from in-patient to out-patient treatment in depression. Deutsches Arzteblatt Int. 2020;117:472-9.

26. Arbeitsgemeinschaft der Wissenschaftlichen Medizinischen Fachgesellschaften-Leitlinie Vorgehen bei Terminüberschreitung und Übertragung. 
https://www.awmf.org/uploads/tx_szleitlinien/0150651_S1_Termin\%C3\%BCberschreitung_\%C3\% 9Cbertragung_02-2014-verlaengert_01.pdf. Accessed June 2021.

27. Kuriya B, Hernández-Díaz S, Liu J, Bermas BL, Daniel G, Solomon DH. Patterns of medication use during pregnancy in rheumatoid arthritis. Arthritis Care Res (Hoboken). 2011;63:721-8.

28. Desai RJ, Huybrechts KF, Bateman BT, et al. Brief report: patterns and secular trends in use of immunomodulatory agents during pregnancy in women with rheumatic conditions. Arthritis Rheumatol. 2016;68:1183-9.

29. Tsao NW, Lynd LD, Sadatsafavi M, Hanley G, De Vera MA. Patterns of biologics utilization and discontinuation before and during pregnancy in women with autoimmune diseases: a populationbased cohort study. Arthritis Care Res (Hoboken). 2018;70:979-86.

30. Palmsten K, Rolland M, Hebert MF, et al. Patterns of prednisone use during pregnancy in women with rheumatoid arthritis: daily and cumulative dose. Pharmacoepidemiol Drug Saf. 2018;27:430-8.

31. Belleudi V, Poggi FR, Perna S, et al. Drug discontinuation in pregnant women with psoriasis: the PSO-MOTHER cohort study. Pharmacoepidemiol Drug Saf. 2020;29:904-12.

32. Tincani A, Taylor P, Fischer-Betz R, Ecoffet C, Chakravarty E. FRI0693 Fears and misconceptions of women with chronic rheumatic diseases on their journey to motherhood. Ann Rheum Dis. 2018;77: 866.

33. Kavanaugh A, Cush JJ, Ahmed MS, et al. Proceedings from the American college of rheumatology reproductive health summit: the management of fertility, pregnancy, and lactation in women with autoimmune and systemic inflammatory diseases. Arthritis Care Res (Hoboken). 2015;67:313-25.

34. Embryotox-Drug safety in pregnancy and lactation. https://www.embryotox.de/. Accessed Sept 2020.

35. Elenkov IJ, Wilder RL, Bakalov VK, et al. IL-12, TNF$\alpha$, and hormonal changes during late pregnancy and early postpartum: implications for autoimmune disease activity during these times. J Clin Endocrinol Metab. 2001;86:4933-8.

36. Chawanpaiboon S, Vogel JP, Moller A-B, et al. Global, regional, and national estimates of levels of preterm birth in 2014: a systematic review and modelling analysis. Lancet Glob Health. 2019;7:e37-46.

37. Meissner Y, Fischer-Betz R, Andreoli L, et al. EULAR recommendations for a core data set for pregnancy registries in rheumatology. Ann Rheum Dis. 2021;80:49-56.

38. Meissner Y, Strangfeld A, Costedoat-Chalumeau N, et al. European Network of Pregnancy Registers in Rheumatology (EuNeP)-an overview of procedures and data collection. Arthritis Res Ther. 2019;21: 241.

39. Thanh BYL, Lumbiganon P, Pattanittum P, et al. Mode of delivery and pregnancy outcomes in preterm birth: a secondary analysis of the WHO Global and Multi-country Surveys. Sci Rep. 2019;9:15556.

40. Jukic AM, Baird DD, Weinberg CR, McConnaughey DR, Wilcox AJ. Length of human pregnancy and contributors to its natural variation. Hum Reprod (Oxford, England). 2013;28:2848-55. 